\title{
COMPUTING PRACTICES
}

\section{Coping with Java Programming Stress}

Programmers who use Java know that it's a good language, but it isn't ideal. Being aware of Java's w eaknesses, like its protected access and constructor confusion, will help you deal with them more intelligently.

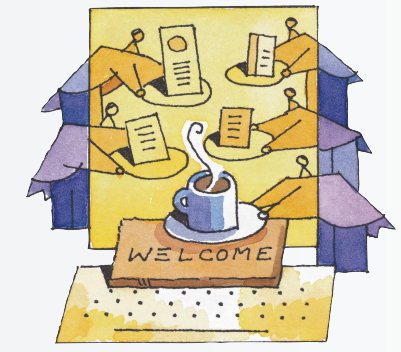

Roger $\mathrm{T}$. Alexander George M ason University

James $M$. Bieman

Colorado

State

University

John Viega

Reliable

Software

Technologies

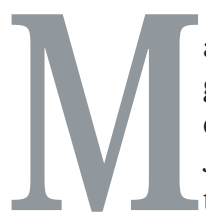

any developers view Java as the language solution to complex software engineering problems. They expect J ava programs to resist system crashes, to be written onceand run everywhere, and to withstand malicious attacks. For the most part, these expectations are reasonable. Java has many attributes that promote reliable, bug-free software: memory management to prevent memory leaks, strong type checking to prevent the misuse of objects, and built-in support for exception handling. Java's virtual machine model increases portability and its security model provides a degree of safety when importing externally developed code. All these features are a great improvement over C ++, J ava's nominal predecessor. Indeed, initial experimental results show greater programmer productivity and fewer program bugs for development with J ava versus $\mathrm{C}++.{ }^{1}$

Unfortunately, however, no language is ideal, and some features of J ava contribute to rather than alleviate programmer stress because they create obscure places for bugs to hide. We have identified seven features that can lead to particularly resistant bugs. 0 ur goal is not to indict J ava-we are strong supporters, and our own organizations have adopted J ava as their primary programming language. Rather, wewant programmers to better understand J ava's weaknesses and know how to cope with them. In somecases, thestrategies we suggest can prevent the weakness from affecting implementation. In other cases, they can minimize the damage. By being aw are of these pitfalls and cop- ing mechanisms, programmers can make sure that Java's design flaws don't make implementation more painful than it has to be.

\section{ILLUSORY PROTECTION}

The term " protected" implies support for encapsulation. When you see it before a program component, such as a variable or method, you naturally assume that visibility to other components is restricted. That is the purpose of encapsulation - to guard the integrity of the protected component or the entity that owns it. O nce components outside that visibility border have access to the protected member, that integrity cannot be guaranteed. This is the case in Java: The visibility hole for members specified as having protected access is so large that protection is merely an illusion. A similar problem occurs for class members not specified as having a particular access (protected, public, or private).

LikeC ++ , in Java " protected" means access to other members of the same enclosing class and to members of its descendants via inheritance. Such access increases the coupling between class definitions, but when an object references a superclass's variable, it is really just referencing part of its own state. J ava supports encapsulation, but it also grants the same access to members of any class in the same package as the class having the protected member. Thus, any class with the same package designator can read and write to protected fields in any other class with the same package designator.

This creates two kinds of undesirable coupling: com- 


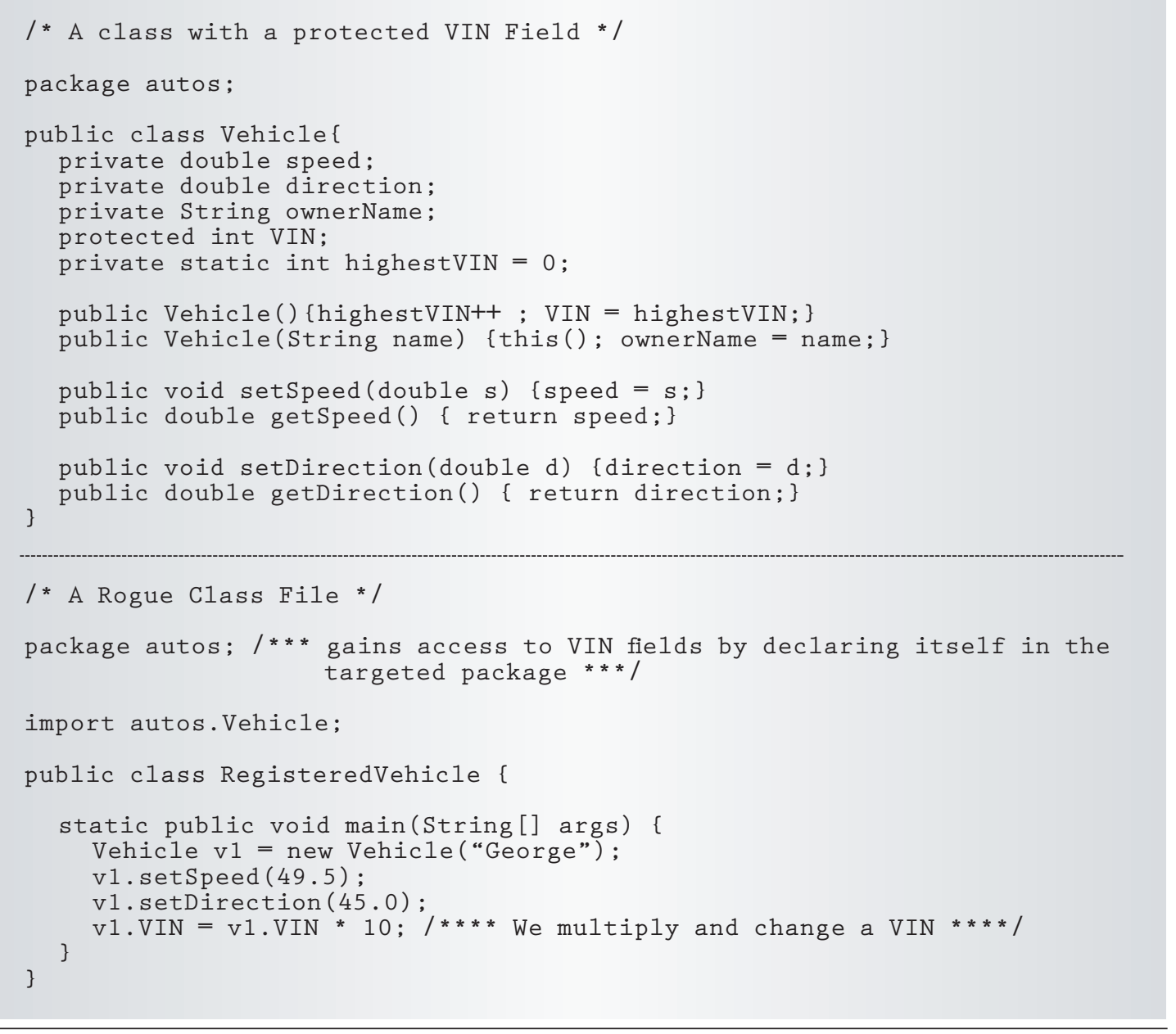

Figure 1. Why protection is weak with Java's protected access. The term "protected" implies support for encapsulation, but in this example, the RegisteredVehicle class breaks the encapsulation of the protected instance variable VIN in the Vehicle class. As a result, the RegisteredVehicle class can circumvent any constraints imposed in VIN by Vehicle and possibly make the state of a Vehicle instance inconsistent. [Example from The Java Programming Language, 2nd ed., $K$. Arnold and J. Gosling, Addison-Wesley, Reading, Mass., 1997]

mon coupling between all objects in the same package that reference a protected instance, and content coupling when objects reference a protected method that implements representation-dependent behavior.

The result is that any change to a protected member can ripple across to an unlimited (and possibly expanding) number of classes with the same package designator. And any component with the same package designator can modify a protected variable and force objects into invalid states.

Figure 1 shows how Java's access rules fail to support encapsulation when a new class is added. In the Vehicle class, the protected instancevariableVIN represents a Vehicle instance or object's vehicle identification number. VIN should be unique for each Vehicle object and should not change during that object's life. These conditions are the V ehicle object's implied variants.

H ow ever, because the R egisteredVehicle class is in the Rogue Class File and is a member of the autos package, it can access the protected variableVIN and possibly modify it, which in turn can violate the implied invariants of the Vehicle object described ear- lier. This object's behavior is now quite unpredictable. Certainly, if used with care, a package can define a collection of closely related abstractions that honor each other's semantics and consistency rules. The point is that Java cannot enforce such practices. You must rely on local honored conventions, such as coding standards, which may fail to prevent inappropriate access.

It is, of course, convenient to be able to add a new class into a package simply by using the package designator in the class code. Unfortunately, this convenience comes at the cost of encapsulation and safety. An arbitrary third party unaware of any established convention or policy could add a class just as easily. $\mathrm{N}$ ew classes added to the same package thus gain complete access to all protected members of every other class in the named package. And these new classes can subsequently violate (inadvertently or deliberately) any conventions or policies.

How to cope. R egrettably, the only way to protect a member from undesired access is to avoid using protected access. Even though you often want descendant classes to access protected members, there is just no way to restrict access to the descendants only. 


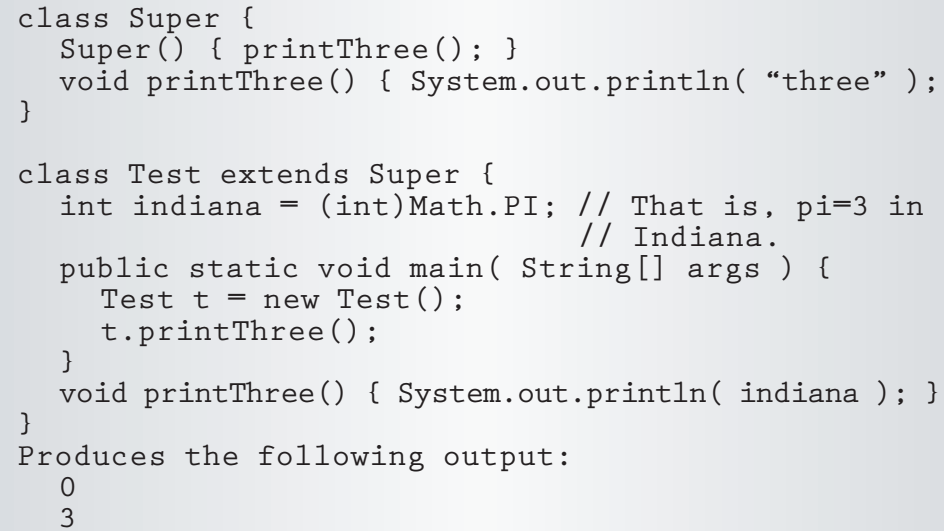

Figure 2. An example of the complex order of initialization and construction that causes constructor confusion. A constructor, Super(), causes an uninitialized variable, indiana, to be used, when the program initializes a subclass, Test. [Example from The Java Language Specification, J. Gosling, B. Joy, and G. Steele, Addison-Wesley, Reading, Mass., 1996, p. 231.]

Until the nature of protected access in J ava changes, we suggest treating protected access as if it reads "unprotected." Make no assumptions about the integrity of any class with protected members.

\section{CONSTRUCTOR CONFUSION}

O ne of J ava's advertised strengths is that it initializes all variables before the program uses them. Thus, in principle, a program will invoke a class's methods only after it has initialized all class instance variables. However, the semantics of initialization and construction in Java are not that simple. For example, a program can use instance variables before it builds the object that owns them.

The confusion results in part from the distinction between variable initialization and class construction and the order in which they can occur. When it creates a new class instance, the program first initializes variables local to that class. It then executes superclass constructors and explicitly initializes any local variables. Finally, it executes the local constructor, if it is present. A constructor can call methods, which the program can override in a descendant class. When a superclass constructor calls an overriding method while the program is building a descendant class object, the overriding method will execute before the program finishes initializing the descendant class instance. Because the construction process has not set thelocal variables that the overriding method can use, strange and unanticipated behavior can result.

Figure 2 demonstrates the complex order of initialization and construction and the ensuing confusion. Thefirst statement in the method main of the Test class creates a new Test object. The program then initial- izes the instance variable indiana to the default value 0 , deferring the explicit initialization to the value of $M$ ath.PI. The program continues by invoking the constructor of the superclass, Super(), which in turn invokes the PrintThree() method. The method invoked is not the PrintThree() method within Super, however, but the PrintT hree() method in the Test class.

The program invokes the method even though it has not completely initialized indiana. Thus, PrintThree() prints a 0, which is indiana's current value. The program then regains control from Super's constructor and initializes indiana to the explicit value $M$ ath.PI (the floating-point value of $\pi$ becomes the integer 3). If the Test class has a constructor, the program would run it now and complete the building of the Test object (t). The program then invokes printThree() of the Test object, which prints out the current value of indiana, now 3.

$M$ ethods that execute before initialization or construction are dangerous at best. Their behavior is likely to invalidate assumptions made by the authors of both the parent and descendant classes. When a base class constructor calls a method, unless the constructor invokes only final methods, the method defined in the base class may not be the one that actually executes. When this happens, the assumptions about the called method aren't likely to hold.

How to cope. O ne approach is to require that all method calls in constructors invoke only local methods designated as final. This will not solve the problem, however, unless all local method calls made from a constructor result in the execution of only methods that are also defined to be final. This makes it extremely difficult to ensure correctness if you are designing a descendant class. You must have a detailed understanding of the semantics of theimplementation of all ancestor classes- particularly how an overriding method affects the parent class's state-space and which methods could possible execute in the unconstructed descendant class object.

This constructor confusion is likely to be the source of many faults, particularly if you have a $\mathrm{C}++$ background, since how $\mathrm{C}++$ constructors deal with local method calls is nearly the opposite of how J ava constructors deal with them. For example, suppose the program is constructing an instance of a derived class in $\mathrm{CH}$. A call from a base-class constructor to a polymorphic (virtual) method defined in the base class always results in the execution of the base-class method, even when the derived class has an overriding definition of the called method. This $\mathrm{C}++$ construction behavior is in stark contrast to that in Java.

\section{FINALIZATION FOLLIES}

Because of J ava's mandated garbage collection, you can ignore the details of memory management. 
Unfortunately, you must still manage the ownership of other resources. Thus, you must deal with many of the complex issues that $\mathrm{C}++$ programmers address using destructors. Although memory leaks will not occur, scheduling the execution of Java class finalizers, ${ }^{2}$ Java's form of destructor, can cause other resource leaks.

Java finalizer methods run when the program is through with an object and must release resources the object still holds. H owever, unless explicitly invoked, a finalizer runs only during garbage collection, rather than when the object loses its last reference. Thus, finalizers run at un predictable times, just like garbage collection. The uncertainty about the time that the finalizer runs can lead to trouble. Suppose a class has a constructor that allocates a netw ork connection and a finalizer that closes down the connection. M any systems map each network connection to a file pointer in the operating system. Generally, relatively few file pointers can be open at once. If a program instantiates and then discards a large number of these objects before the garbage collector calls any finalizers, any attempt to create a new file or network connection will fail.

How to cope. Don't count on finalizers executing in a timely manner. In fact, there is no guarantee that finalizers will ever run at all. For example, when the program exits, no finalizers will run for any objects that have become garbage since the last collection, unless the programmer explicitly ensured that the program called System.runFinalizersO nExit(true). Even that is no guarantee that the finalizer will run. For example, the current version of Sun's J ava virtual machine will not run the finalizer if an outside signal terminates it.

Also, don't expect finalizers to execute in a deterministic order. For example, finalizers will not necessarily run in the order that the objects became garbage; the actual order is unspecified. ${ }^{2}$

The best strategy is to avoid finalization if possible. If you must use it, and your finalizers must be called in a timely manner, explicitly call the garbage collector that will invoke the finalizers. For this to work, you must know beforehand that a given object will be available for finalization, which means that you must track all references to that object. An explicit call to the garbage collector will not invoke an object's finalizer if any references to the object remain.

An alternative is to add public methods that the program can call to release resources an object is holding even though it no longer needs them. H owever, again, you must track all references to the object holding the reso urces and assign the responsibility for calling the methods. This isn't trivial, and an error can be costly: A resource could be deleted when clients are still using it.

\section{INHERITANCE WITHOUT SPECIALIZATION}

Subclasses are descendants of other defined classes. Java and other object-oriented Ianguages let you substitute a subclass object for a superclass object. However, you must satisfy certain properties to guarantee that your substitution is safe. ${ }^{3} O$ ne safe substitution is when the subclass is a specialization of the superclass. For example, a Cartesian point with color attributes can be a specialization of a Cartesian point without color. You can then substitute a colored point for a plain point because any behavior of plain points also applies to colored points.

Problems can occur when a subclass is not a true specialization of its superclass. Consider the java.util.Stack class, which is part of the java.util package. Class java.util.Stack is a subclass of java.util.Vector. Stack defines common stack methods such as push(), pop(), and peek(). H owever, because Stack is a subclass of Vector, it inherits all the methods Vector defines. Thus, you can supply a Stack object wherever the program specifies a Vector object. A program can insert or delete elements at specified locations in a Stack object using Vector's insertElementA t or removeE lementA t methods. It can even use Vector's removeE lement method to remove a specified element from a Stack object without regard to the element's position in the stack.

Consequently, the java.util.Stack can exhibit behavior that is not consistent with the notion of a stack as a last-in, first-out entity. In addition, a program can access all the Vector operations on Stack objects directly when the Stack objects are not being substituted for Vector operations.

A stack is not a specialized vector, and it should not inherit vector operations. Instead, a vector should be a hidden, private representation of a stack. Stack objects cannot then export inappropriate vector operations. This preferred design uses aggregation, which lets you use inheritance and polymorphism to replace the vector representation with alternative implementations. If you use inheritance properly, the design will be more flexible and efficient.

How to cope. In general, substitution will be safe if you use a subclass when the derived class is a specialization of the superclass. In this "is-a" relationship, subclass objects behave similarly to superclass objects but have additional features, operations, or both. If you are unsure how to use inheritance, Bertrand $M$ eyer offers a good taxonomy that classifies both proper and improper uses. ${ }^{4}$ Improper use of subclasses in Java can be an especially troublesome source of bugs that are difficult to diagnose and correct. Java does not provide the mechanisms that $\mathrm{C}++$ does to make improper subclasses a bit safer. In particular,
Improper use of

subclasses in Java

can be an especially

troublesome source

of bugs that are

difficult to diagnose and correct. 


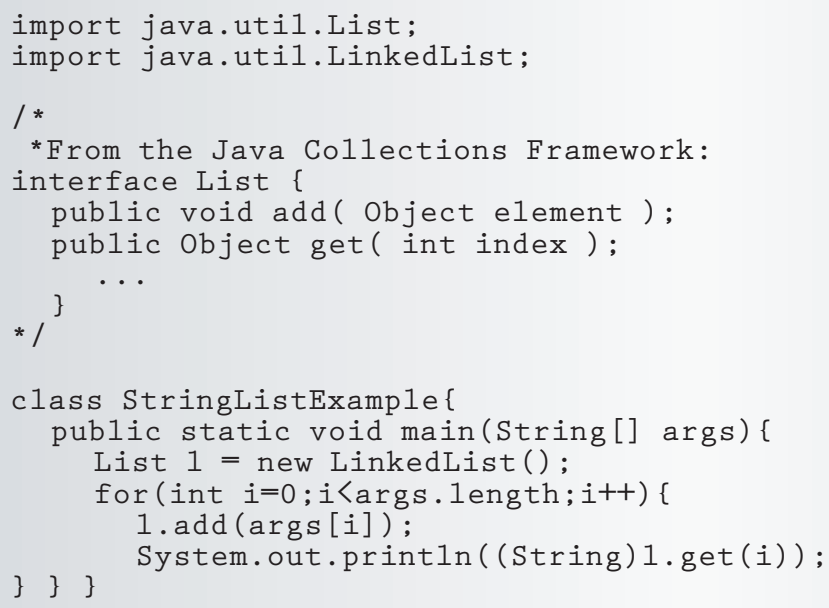

(a)

class StringList \{

\section{(b)}

class RunTimeList

private List my_list;

private Class thisClass;

RunTimeList() \{my_list $=$ new LinkedList();

void add(Object elem) throws BadElement

if (my_list.isEmpty()) thisClass = elem.getClass();

if (thisclass != elem.getClass())

throw new BadElement(thisClass, elem.getClass());

\}

(c)

Figure 3. Three possible strategies for creating an object from the Java Collections Framework class LinkedList, which is meant to be a homogeneous list of String elements. (a) Use a universal list, a list of Object elements, to hold Strings. (b) Create a special-purpose StringList that can hold only String objects. (c) Use a list that sets the class of its contents when the program inserts the first element at runtime. None of these approaches is ideal.

there is no mechanism to hide inherited members or to break the type relationship with the subclass's parent. Thus, there is no way to prevent a client from seeing a descendant as an instance of its base class.

O ne approach is to provide overriding methods for each inherited method and implement them by throwing invalid method exceptions. Unfortunately, you cannot override any methods that are declared as final in a parent class. You must also declare the exception in the parent class unless you throw an unchecked exception, such as those derived from R untimeException. A nother possible solution is to use some sort of assertion mechanism to restrict the use of inappropriate inherited methods.

\section{CONTAINER LIMITATIONS}

J ava provides little flexibility for creating specialized, homogeneous container classes. You must either use containers that can hold anything or writespecialpurpose classes that define containers for each kind of element. Java does not yet support type-safe parameterized classes, as C ++does with templates or Ada does with generics. Instead, it provides the universal base class 0 bject, a superclass to every class.

Suppose you want to create an object from the Java Collections Framework class LinkedList, which is meant to be a homogeneous list of String elements. In $\mathrm{C}++$, you can simply instantiate an object of type LinkedL ist<String>, and the compiler will ensure that 
only String objects are inserted into the LinkedList<String > object. In Java, on the other hand, you must resort to one or a combination of the options in Figure 3:

- Instantiate a LinkedList object that accepts any object whatsoever (objects of class $\mathrm{O}$ bject) and place only objects of class String into it (Figure 3a).

- Write a special-purpose adapter class with the functionality of a LinkedList class that operates only on String objects (Figure 3b).

- Write a LinkedL ist class that, at runtime, sets the type of inserted objects according to the class of the first object inserted (Figure 3c).

How to cope. Unfortunately, there is no workaround. O ur best advice is to weigh the risks of each approach and then proceed with caution. The first approach is the most common, but provides no type safety. You must ensure that the program inserts only objects of the desired class into a list and explicitly puts objects from the list back into the desired class. Because the program must return the objects to the desired classperform casting - at runtime, a method might insert non-String objects into the list. To prevent these kinds of errors, you must track type information.

The second approach ensures that the program can type-check calls to container operations properly at compile time. In addition, the special-purposeclass can perform all casting. Of the three approaches, it offers the most type safety, but it does so at the expense of proliferating nearly identical classes. To minimize code replication, you can have the adapter provide the necessary interface, but implement it in terms of LinkedL ist. This approach imposes the required level of type safety while reusing the existing available implementation.

The advantage of the third approach is that you can implement it using only one class- the class that captures the type of the first object to be inserted into the list. H ow ever, again, you must explicitly cast elements retrieved from the list back into the desired class, so the third approach has similar drawbacks to the first.

\section{NOT-SO-FINAL PARAM ETERS}

In J ava, a method can changethe state of any object of the class that the method is a member of. Thus, to be safe, a client must assume that a method invocation on an object can modify that object's state. If you are programming that client, you must look at the implementation of the called method to really know if the object's state has changed. Unfortunately, because you generally won't have access to the implementation, you must trust the documentation, which, of course, imposes no guarantees or constraints on the called method's implementation.

Your only solution is to document all side effects and ensure that method implementations remain consistent with the documentation. Even this will not work on Java components obtained externally.

Java 1.1 lets you declare a method's formal arguments to be final, ensuring that the state of the argument cannot change. R egrettably, the guarantee applies only to the state of the parameter variable itself, not to the state of any class it references. Java does not let you change the value of a formal argument that is a reference to an object even though that argument is declared to be final, but it does let you change the state of the object being referenced. You can use the final parameter variable to invoke any method defined in the object's class. Thus, when a program supplies an object reference as an argument to a method call, the state of the referenced object argument can change, even if the associated formal parameter is designated as final. You are forced to trust the called method, inspect the method if possible, or add code to verify that no state changes have occurred and add errorhandling code.

The protection the final parameter designator provides has severe limitations that could cause a system to enter an inconsistent state. Testing alone cannot guarantee that all methods behave as expected.

How to cope. Your only protection is to make sure that methods contain correct documentation that explicitly describes the effects of a particular method call. These effects include both those on a given instance and those on any instances passed via object references as actual arguments.

\section{INITIALIZATION DIFFUSION}

The JDK 1.1 Java Language Specification includes code blocks that initialize the state of object instances, which are similar to blocks that initialize class state. You can write an instance initialization block simply as an unlabeled block of code that appears at any location in a class definition. There can be multiple instance initialization blocks, possibly distributed at various locations within a class. The program executes initialization blocks in the order they appear in a class.

Figure 4 demonstrates how initialization blocks diffuse initialization across a class. When the program creates a new Vehicle (or VehicleD iffusion) object, we want to increment the static variable highestV IN so that we can use a unique VIN for each new Vehicle object. Class Vehicle updates highestVIN in the expected place-within the constructor method Vehicle(). Class VehicleD iffusion updates highestV IN in an initialization block rather than in a constructor. To further confuse code readers, the program places the update in a location apart from the constructors.
The protection the

final parameter

designator provides

has severe

limitations that

could cause a

system to enter an

inconsistent state. 
write software components that depend on this implementation, which violates encapsulation principles.

$M$ aking code easy to understand is key to maintenance and reuse. Programmers writing a client must now wade through an entire class body just to view the public interface of a potential server class. The solution to this problem would not require separate interface and implementation files for each class, as the.$h$ and .cpp files in C ++. R ather, J ava could have a separate syntactic mechanism within a class that specifies its interface. The corresponding implementation might still appear in line within the class body.

The Javadoc facility can extract interface information. H owever, Javadoc relies on a class's author to w rite appropriate J avadoc comments and keep them current. Comments and code usually diverge over time, however, which means that the Javadoc interface information will eventually be inaccurate.

No support for asser tions. O bject-oriented languages, such as Eiffel and Clu, support assertions. ${ }^{7,8}$ Properly used, an assertion mechanism can increase a component's quality and correctness. Programmers can specify pre- and postconditions for methods and data invariants for class state variables.

Java does not have a built-in assertion mechanism, although tools are freely available for assertion support, such as Reliable Software Technologies' A ssertM ate ${ }^{9}$ and Reliable Systems' iC ontract. ${ }^{10}$ The Java FAQ Web site ${ }^{2}$ tells you how to construct a rudimentary assertion mechanism. Eventually, wehope to see J ava support its own assertion mechanism.

Ar $r$ ay type-checking failur es. Static type-checking limitations can allow programs with obscure array type errors to compile. Although dynamically binding subclass objects is usually type-safeand flexible, it can lead to a type clash or covariance problem if subclass objects are bound with arrays. Generally, you can supply an instance that is a subclass of the declared type of the formal parameter as an argument in a method call. $\mathrm{H}$ ow ever, sometimes dynamic binding conventions and asso ciated type-checking rules cannot detect type errors.

Figure 5 shows why. In this program, all actual parameters are instances of the formal parameters' subclasses. The program compiles even though it contains a serious type error. $\mathrm{N}$ o type errors are detected during compilation because argument anA rray $\mathrm{fB}$ is an instance of a subclass of formal parameter $x$ 's class, $A[]$, and the type of argument a exactly matches the type of formal parameter $y$. Yet, the program fails at runtime.

T he shortcomings we have identified are worrisome because J ava is intended for the development of concurrent, distributed, and critical systems. O ur coping suggestions can remedy or soften the effects of these problems or help you avoid them,

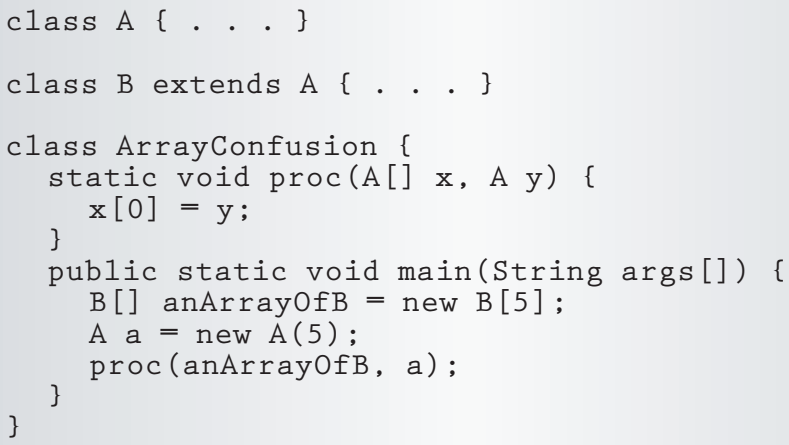

Figure 5. A sample array type-checking problem. ArrayConfusion. main calls ArrayConfusion. proc with two arguments: anArrayOfB and a, an instance of class A. ArrayConfusion. proc has two formal parameters, $\mathrm{x}$, an array of A objects, and $\mathrm{x}$, an A object. Java's static type-checking does not catch the type error when the first argument to ArrayConfusion. proc is an array of class $B$ and the second argument is an object of class $A$. The assignment $\mathrm{x}[0]=\mathrm{y}$ raises a runtime exception. The assignment $\mathrm{x}[0] \mathrm{y}$ y raises a java. lang. ArrayStoreException because an array of class B objects cannot store an object of class $A$. A is not a subclass of $B$. This program has an illegal assignment of a supertype to a subtype variable and fails at runtime, yet it compiled with no detected errors.

but changes to the language itself would offer a more effective long-term solution. H ere's our wish list:

- Replacethe package-level component of protected access with a mechanism that lets a class specify what other specific classes, or group of classes with a particular characteristic, can access it.

- M ake default access private.

- Changethe semantics so that constructors cannot invoke any methods, directly or indirectly, in subclasses.

- Support templates or generics.

- M akethe final parameter designator ensure that a method with a final parameter cannot modify the state of objects the parameter references. Add

\section{A Coping Checklist}

$\checkmark$ Avoid using protected or package-level (default) access; declare all members as either private or public.

$\checkmark$ Take extra care in understanding the construction of new objects that override superclass methods and instance variables.

$\checkmark$ Use container classes with caution. Java type checking is not effective here.

$\checkmark$ Use the subclassing mechanism only to define specializations of a superclass.

$\checkmark$ Explicitly force finalizers to run when you want them to.

$\checkmark$ Use inheritance only to model is-a relationships.

$\checkmark$ D ocument all side effects, and make sure that the documentation is consistent with the code.

$\checkmark$ Avoid using instance initializer blocks. 
a constant method designator to ensure that the state of the object containing the method does not change.

- Require a keyw ord to specify an instance initializer and allow only one such initializer block per class.

It would be unrealistic to expect future versions of Java to incorporate all these changes. A language many people use must satisfy myriad, often competing, interests. Some of our changes could be made with little effort, but others will require major effort and probably some research.

An immediate first step would be to use testing and static program analysis to identify programs with the problems we've described. For example, static analysis can easily identify programs that use instance initializer blocks, protected and package (default) access, or both. A nother immediate goal is to develop tools and techniques, through either static analysis or testing, that can identify programs suffering from the J ava weaknesses we have identified.

\section{Acknowledgments}

Wethank R eliable Software Technologies, Sterling, Va., and the University of M aryland at College Park for their generous support during Jim Bieman's sabbatical when we prepared this article. We also thank J efi $O$ fiutt and Gary M cGraw, whose comments on earlier drafts greatly improved the presentation. Finally, we thank the anonymous Computer reviewers for their comments, which greatly improved both content and presentation.

\section{References}

1. G. Phipps, "Comparing O bserved Bug and Productivity Rates for Java and $\mathrm{C}++$," Software: Practice and Experience, A pr. 1999, pp. 345-358.

2. P. van der Linden, "Frequently Asked Q uestions (with Answers) for Programmers Using the Java Language," http://www.afu.com/ javafaq.html.

3. G. Leavens, "M odular Specification and Verification of O bject-O riented Programs," IEEE Software, N ov./Dec., 1991, pp. 72-80.

4. B. M eyer, "The M any Faces of Inheritance: A Taxonomy of Taxonomy," Computer, M ay 1996, pp. 105-108.

5. K. A rnold and J. Gosling, The Java Programming L anguage, 2nd ed., Addison-Wesley, R eading, M ass., 1997.

6. H. Thimbleby, "A Critique of J ava," Software: Practice and Experience, M ay 1999, pp. 457-478.

7. B. Liskov and J. Guttag, A bstraction and Specification in Program D evelopment, M IT Press, Cambridge, M ass., 1986.
8. B. M eyer, Eiffel: The L anguage, Prentice $\mathrm{H}$ all, Upper Saddle River, N .J ., 1992.

9. J. Payne, M. Schatz, and M. Schmid, "Implementing Assertions for Java," D r. D obb's J., Jan. 1998, http:// www.ddj.com/articles/1998/9801/9801d/9801d.htm\#rel

10. R. K ramer, "iC ontract-The Java Design by Contract Tool," Proc.Technology of $O$ bject- $O$ riented $L$ anguages and Systems, (TO O L S-28, 98), IEEE CS Press, LoS Alamitos, Calif., 1998, pp. 295-307.

Roger T. Alexander is a member of the research faculty at G eorge M ason U niversity and a principal member of the technical staff at the Softw are Productivity Consortium. $\mathrm{H}$ is research interests include object-oriented software testing, architecture testing, specification-based testing, software reliability, and software metrics. $\mathrm{H}$ e received an $\mathrm{MS}$ in software engineering from G eorge M ason U niversity, where he is a PhD candidate in computer science. Alexander is a senior member of the IEEE. Contact him at rtalexander@ computer.org or ralexand@gmu.edu.

James M. Bieman is an associate professor of computer science at Colorado State U niversity. $\mathrm{H}$ is current research interests are evaluating object-oriented designs and developing ways to quantify design attributes in terms of architectural structures and patterns. $\mathrm{He}$ is also studying the relationship betw een design attributes and external quality attributes such as maintainability, testability, and reliability. Bieman received a PhD in computer science from the $U$ niversity of Southwestern L ouisiana. H e is a senior member of the IEEE and is chair of the Steering Committee for the IEEE-CSI nternational Symposium on SoftwareM etrics. Contact him at bieman@cs.colostate.edu.

John Viega is a senior research associate and consultant at R eliable Software Technologies. $\mathrm{H}$ e is the principal investigator on a DARPA-sponsored grant for developing security extensions for standard programming languages. H ealso writes a bimonthly column on software security assurance for developers (http://www.ibm.com/developer/security). Viega received an M S in computer science from the U niversity of Virginia. Contact him at jviega@rst.com. 